\title{
Two Methods of Mechanical Noise Reduction of Recorded Speech During Phonation in an MRI device
}

\author{
J. Přibil ${ }^{1}$, J. Horáček ${ }^{2}$, and P. Horák ${ }^{3}$ \\ ${ }^{1}$ Institute of Measurement Science, SAS, Dúbravská cesta 9, SK-841 04 Bratislava, Slovakia, umerprib@savba.sk \\ ${ }^{2}$ Institute of Thermomechanics, AS CR, v.v.i., Dolejškova 5, CZ-182 00 Prague, Czech Republic \\ ${ }^{3}$ Institute of Photonics and Electronics, AS CR, v.v.i., Chaberská 57, CZ-182 51 Prague, Czech Republic
}

\begin{abstract}
The paper presents two methods of noise reduction of speech signal recorded in an MRI device during phonation for the human vocal tract modelling. The applied approach of noise speech signal cleaning is based on cepstral speech analysis and synthesis because the noise is mainly produced by gradient coils, has a mechanical character, and can be processed in spectral domain. Our first noise reduction method is using real cepstrum limitation and clipping the "peaks" corresponding to the harmonic frequencies of mechanical noise. The second method is coming out from substation of the short-time spectra of two signals recorded withal: the first includes speech and noise, and the second consists of noise only. The resulting speech quality was compared by spectrogram and mean periodogram methods.
\end{abstract}

Keywords: speech processing, noise reduction, NMR imaging

\section{INTRODUCTION}

$\mathrm{B}$ ETTER KNOWLEDGE of the inner structures of human body is possible by using CT or NMR investigations. The non-invasive magnetic resonance scanning of the vocal tract volume of subjects for speech configuration or in phonation position of their resonant cavities for each vowel enables to develop the threedimensional (3D) computer models of the vocal tract. The primary volume models of the human acoustic supraglottal spaces created from the MR images can then be transformed into the 3D finite element (FE) models [1]. The FE models open new possibilities in simulating and understanding production of human voice and speech. The FE models of the human vocal tract enable to perform numerical simulations of voice production. The frequency modal characteristics of the up to now frequently used onedimensional (1D) models showed that the 1D models can closely replicate the behaviour of the $3 \mathrm{D}$ vocal tract only up to about $3 \mathrm{kHz}$. The developed FE models can be useful for studying the influence of vocal tract alterations on acoustic voice quality.

While most of the acoustic energy of the human voice and speech is contained in the frequency range between $70 \mathrm{~Hz}$ to $5 \mathrm{kHz}$, high-fidelity simulation of human voice and speech aims at producing sounds within the whole audible frequency range, i.e. $20 \mathrm{~Hz}-20 \mathrm{kHz}$. Consequently, it is important to develop models that are able to simulate the acoustic properties of the vocal tract with a high accuracy. These models should allow simulating pathological changes or voice quality variations due to slight geometry modifications of the human supraglottal acoustic space.

The purpose of future studies is to develop computational models of the human vocal tract that allow more accurate representation of the $3 \mathrm{D}$ wave propagation, and especially real-time numerical simulations of phonation useful in modelling real clinical situations. Examples are the influence of various inborn defects in human supraglottal spaces (such as velopharyngeal insufficiency or clefting [2]) on speech and voice, or simulations of various post-surgical states in patients, e.g., after tumour extraction in mouth cavity or tonsillectomy [3]. The FE modelling enables also to simulate the influence of the acoustic impedance changes of the vocal tract by phonating into glass tubes or straws used in voice training and therapy in clinical practice [4], [5]. Another application of the FE modelling of phonation is simulation of the so-called "formant tuning" and "singer's or speaker's formant" techniques that belong to the basic qualities of the operatic singers' and voice professionals', which are formed by clustering higher acoustic resonances [6], [7]. However, the proper description is very complex and has not been properly and fully mapped yet. The quality of the developed FE models has to be checked by a sufficiently accurate numerical simulation of the subject's phonation and therefore simultaneous acoustic recording of the subject's voice during the scan procedure is very important.

Usually, in case of whole-body NMR imaginer (MRI), the scanning room presents quite a challenging sound recording environment. Therefore, special solutions for speech signal recording must be realized. One possible way of implementation is the construction of a special sound collector [8], [9], but there is a problem with the requirement of its realization as a completely passive metalfree element without moving parts - because it does not cause any artefacts in the scanning image due to magnetic field homogeneity. A different solution was applied in practice - speech signal collection by optical arrangements using special fibre optical microphones located in the scan room [10]. This approach allows real-time processing of speech signal together with obtaining NMR images, but the practical application is significantly more complicated (synchronization of both processes, special hardware for MRI device, etc.), and more expensive. On the other hand, the cheaper type of optical microphone has the frequency response in the range of $<50 \div 4000 \mathrm{~Hz}>$, which is not sufficient for our intentions. 
There exist several approaches to reduce the noise in speech. One group of these speech enhancement methods is based on spectral subtraction of the estimated background noise [11]. The noise estimation techniques, usually coming out on statistical approaches [12], were not able to track the real variations in the noise, thereby resulting in an artificial residual fluctuating noise and distorted speech. Other noise estimation techniques performed relatively better for stationary and slowly varying noise but showed degradations when the noise was non-stationary. For that reason we use another approach, based on cepstral speech modelling.

\section{SUBJECT \& METHODS}

\subsection{REDUCTION OF GRADIENT COIL NOISE IN SPEECH SIGNAL USING THE CEPSTRAL MODEL}

Contrary to other speech analysis methods (such as LPC), the cepstral speech analysis is performed in the frequency domain. The cepstral speech synthesis (reconstruction of speech signal) is realized by a digital filter implementing approximate inverse cepstral transformation. For voiced speech the filter is excited by a combination of an impulse train and high-pass filtered random noise, for unvoiced speech the excitation is formed by a random noise generator - see Fig.1. The transfer function of the vocal tract model is approximated by Padé approximation of the continued fraction expansion of the exponential function. The error of the inverse cepstral approximation depends on the number and the values of applied cepstral coefficients and the used approximation structure [13].

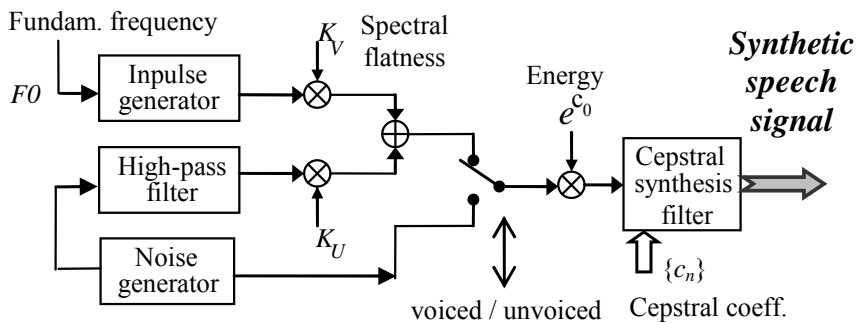

Fig.1. Structure of the cepstral speech synthesizer with mixed excitation.

The fundamental frequency (F0) of voiced speech is represented by a typical peek in the real cepstrum, as well as the mechanical frequencies of gradient coils producing the noise of the MRI machine. Our first noise reduction method is based on the limitation of the real cepstrum and clipping the "wrong peaks" corresponding to the harmonic frequencies of the mechanical noise. The second approach is based on the subtraction of the short-time spectra from two parallel processed signals: from the first microphone including the speech and noise, and from the second microphone consisting only of noise.

\subsection{ONE CHANNEL NOISE REDUCTION BY CEPSTRUM CLIPPING (NOISE REDUCTION METHOD I)}

Cepstral analysis of speech and noise signal is performed in the following way: first the complex spectrum using FFT algorithm is calculated from the input samples (after segmentation and weighting by a Hamming window). In the next step, the power spectrum is computed and the natural logarithm is applied - see the block diagram in Fig.2. Application of inverse FFT algorithm gives the symmetric real cepstrum. By limitation to the first $N_{0}+1$ coefficients, the Z-transform of the real cepstrum can be obtained

$$
C(z)=c_{0}+c_{1} z^{-1}+c_{2} z^{-2}+\ldots+c_{N_{0}} z^{-N_{0}} .
$$

The truncated cepstrum represents an approximation of a log spectrum envelope

$$
E(f)=c_{0}+2 \sum_{n=1}^{N_{0}} c_{n} \cos (n \cdot 2 \pi f),
$$

where the first cepstral coefficient $c_{0}$ corresponds to the signal energy. The whole algorithm works in four steps:

1) calculation of real cepstrum, pitch-period detection, F0 calculation

2) determination of position of peaks in cepstrum corresponding to the frequencies of mechanical noise and minimum number $N_{0}$ of cepstral coefficients (for sufficient log spectrum approximation [13])

3) limitation of real cepstrum and clipping peaks

4) reconstruction of input signal by the pitch-synchronous cepstral speech synthesizer.

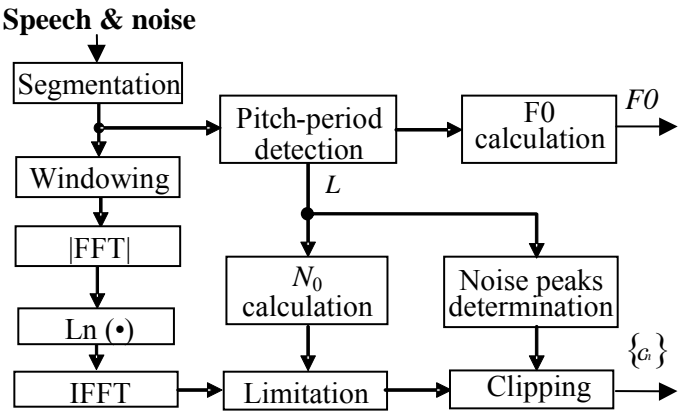

Fig.2. Block diagram of cepstral analysis of the speech and noise signal.

\subsection{TWO CHANNEL NOISE REDUCTION BY SPECTRAL SUBTRACTION (NOISE REDUCTION METHOD II)}

Generally, the noisy speech signal $x(k)$ is interpreted as an addition of a clean speech signal $s(k)$ and an additive noise $n(k)$

$$
x(k)=s(k)+n(k) \text {. }
$$

The noisy signal is segmented and windowed to obtain a short time-frame of noisy speech. By transformation to the frequency domain using the FFT, we get the short-time spectrum $X(f, n)$. The enhanced speech spectrum can be obtained by subtracting a noise magnitude spectrum (loaded by the microphone Mic. 2) from the noisy speech magnitude spectrum (of signal loaded by the first microphone Mic. 1)

$$
S(f, n)=(|X(f, n)|-|N(f, n)|) \cdot e^{\varphi_{n}(f, n)},
$$


where $e^{\varphi_{n}(f, n)}$ represents the phase of noisy spectrum, and $\mathrm{n}$ is index of processed frame. The natural logarithm and IFFT are applied on the resulting spectrum, whereby the limited real cepstrum is obtained next - see block diagram in Fig.3. The final clean signal is reconstructed also by cepstral speech synthesizer.

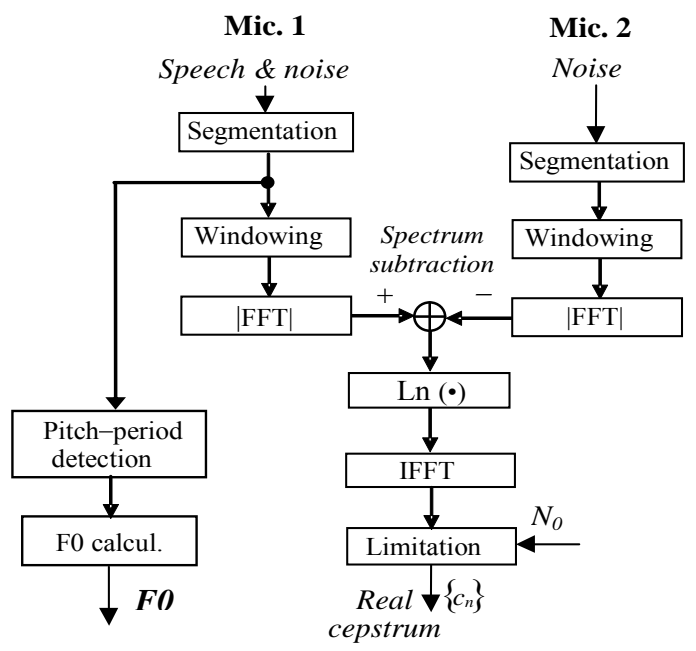

Fig.3. Block diagram of two channel spectral subtraction methods and cepstral analysis.

\subsection{COMPARISON OF NOISE REDUCTION METHODS BY SPECTROGRAMS AND MEAN PERIODOGRAMS}

A spectrogram can be successfully used for visual quality comparison of different approaches to noise reduction on speech. However, results of visual comparison of the whole spectrograms depend strongly on the person that makes this matching. To obtain more precise matching results it is necessary to select typical or interesting parts - the ROI areas. From the chosen ROI area, the mean periodogram calculated by the Welch method [14], [15] can be determined - see block diagram in Fig.4.

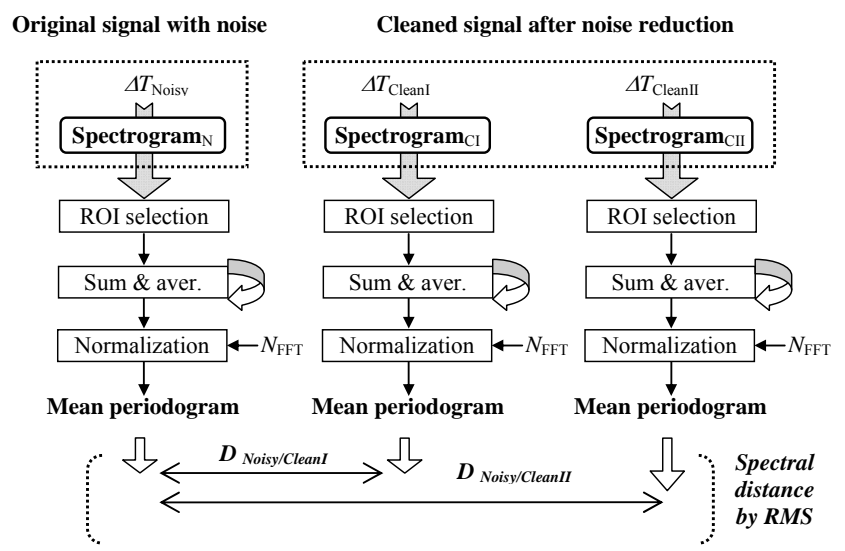

Fig.4. Block diagram of the averaged Welch's periodogram calculation of noisy and cleaned speech signal.

The resulting Welch's periodogram in $[\mathrm{dB}]$ can be used for subsequent comparison. For exact numerical comparison (objective matching method) it is possible to calculate the spectral distance $D_{\text {RMS }}$ (by the RMS method) between different periodograms (between noisy and cleaned speech signal). From periodograms it is also possible to determine the first formant $\mathrm{F} 1$ and $\mathrm{F} 0$ position, and the frequency $F_{\mathrm{N}}$ corresponding to harmonic noise - in this point the spectral difference effected by the noise suppression can be calculated.

\section{EXPERIMENTS AND RESULTS}

Unlike the standard whole-body MRI machine, where the noise is produced only by gradient coils into the scanning tube, in our open-air imaginer, 0.178T imaginer Esaote OPERA [16], the noise is also produced by temperature stabilizer of the MRI device. This noise has almost constant the noise pressure level (NPL) as an equivalent of sound pressure level (SPL) and consequently it can be easily subtracted as a background. Because the noise depends on the position of the measuring microphone, the directional pattern of the noise source in the MRI scanning area must be measured first. After that, the one- and two-channel speech and noise recording experiments were performed.

\subsection{MEASUREMENT OF NOISE PRESSURE LEVEL DISTRIBUTION INTO MRI SCANNING AREA}

The MRI device also includes an adjustable bed which can be positioned in the range of $180 \mathrm{deg}$. (originated from left corner near the temperature stabilizer device). The measuring microphone of the sound level meter was placed at the distance of $60 \mathrm{~cm}$ from the central point of the scanning area representing the receiving coil with the testing phantom (see item 5 in Fig.5). For each of the bed positions, measurement of NPL is realized for the sound level meter located in three heights:

a) basic level (level with the bottom gradient coil),

b) $10 \mathrm{~cm}$ above the bed (in the middle between both coils),

c) $20 \mathrm{~cm}$ above the bed (on level with the upper coil).

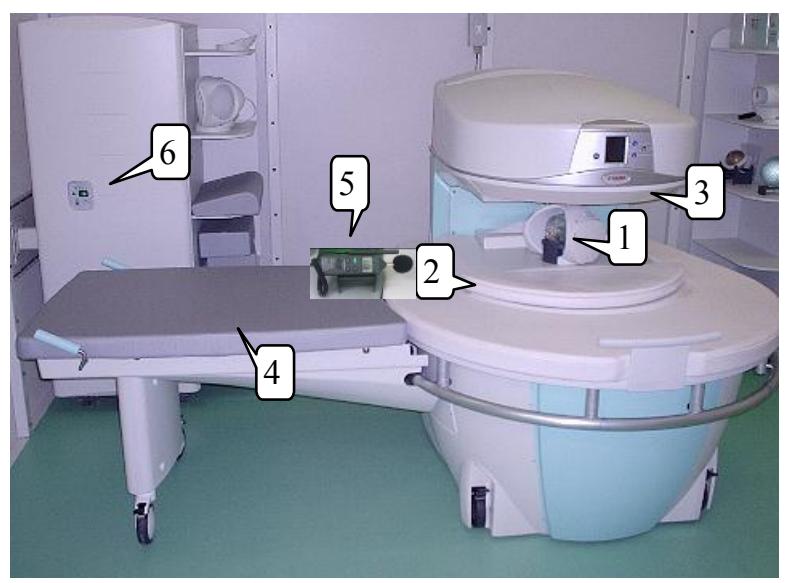

Fig.5. Photo of NMR imaginer OPERA: (1) - middle point of scanner with testing phantom and receiving coil, (2) - lower permanent magnet and gradient coil, (3) - upper permanent magnet and gradient coil, (4) - adjustable bed (in 0 deg. position), (5) sound level meter, (6) - NMR temperature controller.

The separate measurement of background noise (on the bed level height) was subsequently performed. The 
measurement of gradient coil noise pressure level was realized with the following scanning parameters: Gradient Echo sequence $\mathrm{T} 1^{1}$, coronal, with knee scanning coil filled by a spherical testing phantom, with the help of the measuring device DT-8820.

Resulting measured NPL characteristics together with background level $I_{0}$ curve for three microphone heights are shown in Fig.6, the summarized values in numerical form are stored in Table 1.

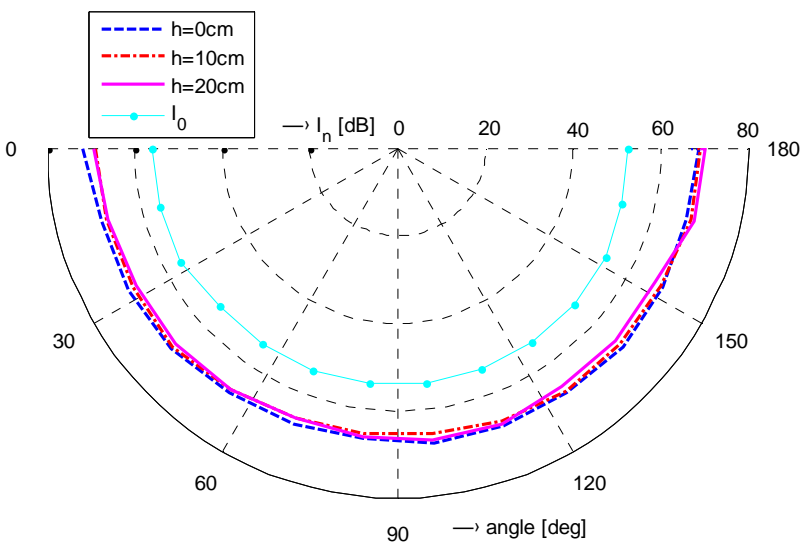

Fig.6. Resulting measured NPL characteristics for three microphone heights together with background level $I_{0}$.

\begin{tabular}{|c|c|c|c|}
\hline \multirow{2}{*}{$\begin{array}{c}\text { Microphone } \\
\text { high [cm] }\end{array}$} & \multicolumn{3}{|c|}{$\mathbf{I}_{\mathbf{N}}[\mathbf{d B}]$} \\
\cline { 2 - 4 } & minimum & maximum & mean \\
\hline 0 & 66.80 & 71.80 & 68.37 \\
\hline 10 & 65.70 & 69.00 & 67.77 \\
\hline 20 & 66.00 & 70.20 & 67.81 \\
\hline
\end{tabular}

Table 1. Summarized values of noise pressure level $I_{\mathrm{N}}$ for three microphone heights.

\subsection{SPEECH AND NOISE RECORDING AND PROCESSING EXPERIMENT}

Our arrangement of speech and noise recording measurement was the following: the bed with the testing person was set to $60 \mathrm{deg}$. position - see Fig.7. For the speech and gradient coil noise signal recordings, the front microphone (Mic. 1) was located on the 150 deg. position; for recording the noise signal only, the back microphone (Mic.2) was placed at the $30 \mathrm{deg}$. position. Both microphones were located $10 \mathrm{~cm}$ above the bed (in the middle between both coils), and in the $60 \mathrm{~cm}$ radius of the central point of scanning area (RF coil). The measurement was realized with the same scanning sequence as in the case of noise distribution experiment; the head of the testing person was inserted into the knee coil (see item 3 in Fig.7), the background noise level (generated mainly by a temperature stabilizer) was $I_{0}=55 \mathrm{~dB}$.

Speech as well as noise was recorded with the help of the M-Audio FireWire 1814 equipment connected to a personal

${ }^{1}$ With internal settings of MRI Opera device parameters [16]: FOV 200x200x 192, 26 slices, slice thickness $=1.8 \mathrm{~mm}$, resolution $=256 \times 256, \quad \mathrm{TR}=500 \mathrm{~ms}, \quad \mathrm{TE}=18 \mathrm{~ms}, \quad$ number of averages $=1$ computer through a high-performance, high-resolution multi-channel interface of the IEEE 1394 (FireWire) bus. As Mic. 1, the professional 1" Behringer dual diaphragm condenser microphone B-2 PRO (with cardioid pickup pattern) was used. For Mic.2, the RØDE NTK 1" condenser microphone with cardioid directional pattern was chosen. Signals from both microphones were originally recorded at $32 \mathrm{kHz}$, and resampled to $16 \mathrm{kHz}$. Collected database of speech and noise signals consists of 90 records of five separately phonated long vowels "a", "e", "i", "o", and "u" from three male and three female non-professional speakers with time duration interval from 8 to $15 \mathrm{sec}$. The frame length depends on the mean pitch period $L_{0}$ of the processed signal. In our experiment, we used 24-ms frames for male voice, and 20-ms frames for female voice. The parameter $N_{0}$ for limitation of real cepstrum was chosen in correspondence with the period of noise part of signal $L_{n}$ equal to 256 (when $N_{\mathrm{FFT}}=1024$ ) for both voices; the minimum-phase cepstral coefficients [12] were subsequently used for signal reconstruction - see Fig. 8 .

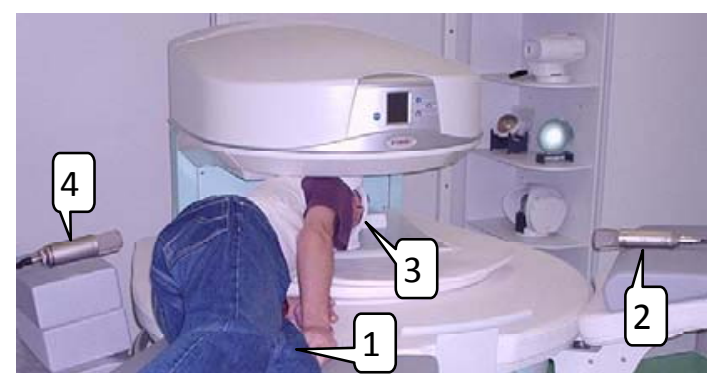

Fig.7. A speech and noise recording experiment arrangement: bed with testing person (1), front read microphone 1 (2), patient's head in RF coil (3), back read microphone 2 (4).
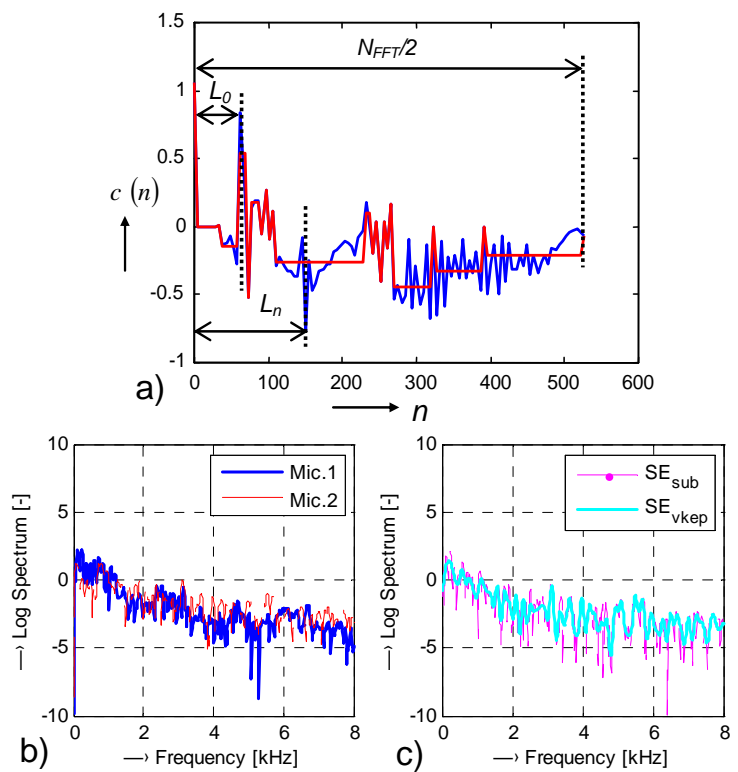

Fig.8. Graphic examples of applied noise reduction method: real cepstrum clipping and limitation of signal recorded by Mic. 1 (a), two $\log$ spectral envelopes of signals recorded by Mic. 1 and Mic. 2 (b), resulting envelope after subtraction and cepstral reconstruction (c); processed speech and noise signal of long vowel "a" $\left(225^{\text {th }}\right.$ frame of 876$)$, female voice $(\mathrm{F} 0 \approx 220 \mathrm{~Hz})$. 


\subsection{COMPARISON BY SPECTROGRAMS AND WELCH'S PERIODOGRAMS}

From the main speech signal databases of long vowels, the derived one, consisting of manually selected ROIs corresponding to the stationary part of the vowels "a", "e", "i", "o", "u", was consequently created. From every long vowel file, 3 to 5 parts with mean duration of $3 \mathrm{sec}$ were selected. Finally, this database consists of 321 items for both voices in total. Since the collected speech material originates from speakers with different mean F0 value (see Table 2), different parameter settings for Welch's periodogram calculation (working window length $L_{W}$ and window overlapping $L_{O}$ ) [14] must be applied. Also the lengths of selected ROI areas were chosen as multiples of basic $L_{\mathrm{W}}$ length setting.

\begin{tabular}{|c|c|c|c|c|c|c|}
\hline & $\mathbf{S 1}_{\mathbf{m}}$ & $\mathbf{S 2}_{\mathbf{m}}$ & $\mathbf{S 3}_{\mathbf{m}}$ & $\mathbf{S 1}_{\mathbf{f}}$ & $\mathbf{S 2}_{\mathbf{f}}$ & $\mathbf{S 3}_{\mathbf{f}}$ \\
\hline $\mathbf{F 0}_{\text {mean }}[\mathbf{H z}]$ & 133 & 127 & 98 & 228 & 177 & 207 \\
\hline
\end{tabular}

Table 2. Speaker mean F0 values - male and female voice.

The comparison with the help of spectrograms and Welch's periodograms was practically performed in four steps:

1) visual comparison and matching of displayed spectrograms (see demonstration examples in Fig.9 and Fig.11),

2) visual comparison of calculated Welch's periodograms (for selected ROI areas),

3) numerical matching of results from the calculated spectral distances between corresponding periodograms by the RMS method (see summary periodogram examples in Fig.10a) and Fig.12a),

4) determination of $\mathrm{F} 1, \mathrm{~F} 0$, and $F_{\mathrm{N}}$ positions, calculation of spectral difference $\Delta \mathrm{P}$ in $[\mathrm{dB}]$ on the $F_{\mathrm{N}}$ position (see details in Fig.10b) and Fig.12b).

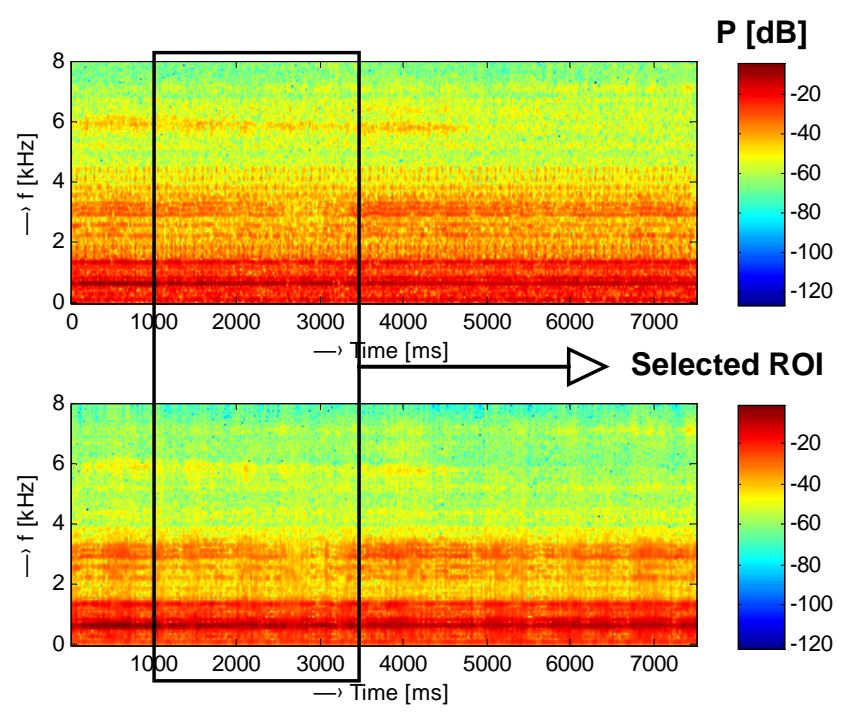

Fig.9. Spectrograms of the processed long vowel "a" (female voice, $\mathrm{F} 0 \approx 230 \mathrm{~Hz}, f_{\mathrm{s}}=16 \mathrm{kHz}$ ): original speech and noise signal (top), reconstructed signal after cepstrum limitation and clipping (bottom).
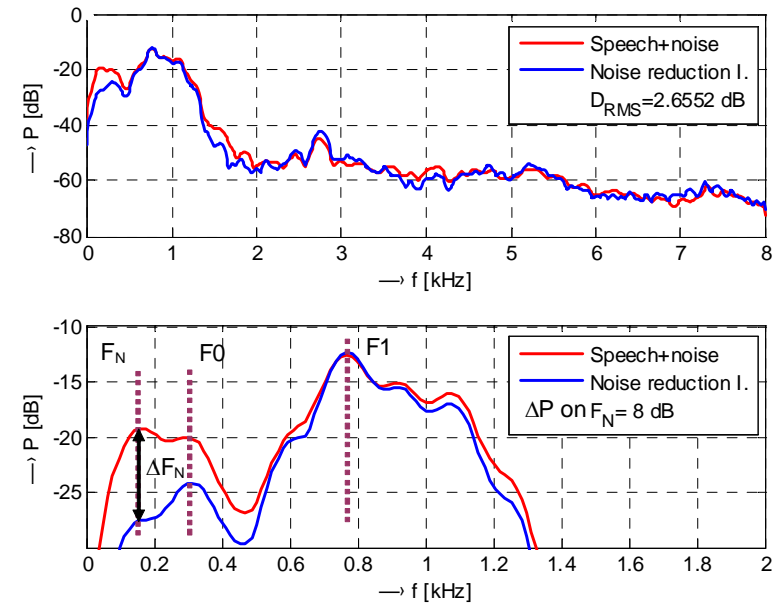

Fig.10. Welch's periodograms in $[\mathrm{dB}]$ of selected ROI from $1 \mathrm{~s}$ to $3.5 \mathrm{~s}$ of the speech signal used for spectrogram calculation in Fig.9: summary periodogram for full frequency range (top), detailed one with determined $F_{\mathrm{N}}, \mathrm{F} 0$ and $\mathrm{F} 1$ positions (bottom).

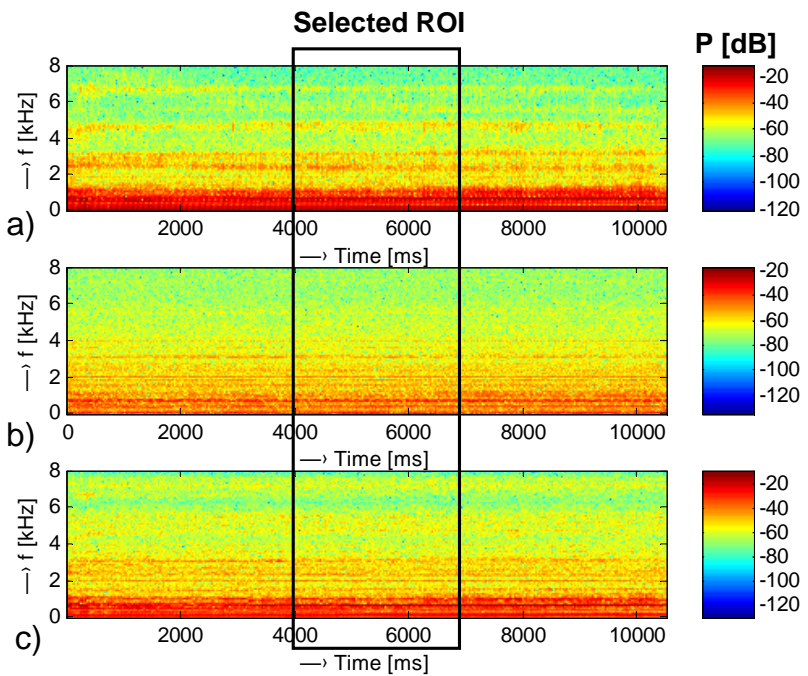

Fig.11. Spectrograms of processed long vowel "o" (female voice, $\mathrm{F} 0 \approx 210 \mathrm{~Hz}, f_{\mathrm{s}}=16 \mathrm{kHz}$ ): speech and noise signal - Mic. 1 (a), noise signal only - Mic. 2 (b), reconstructed signal after spectral envelope subtraction (c).
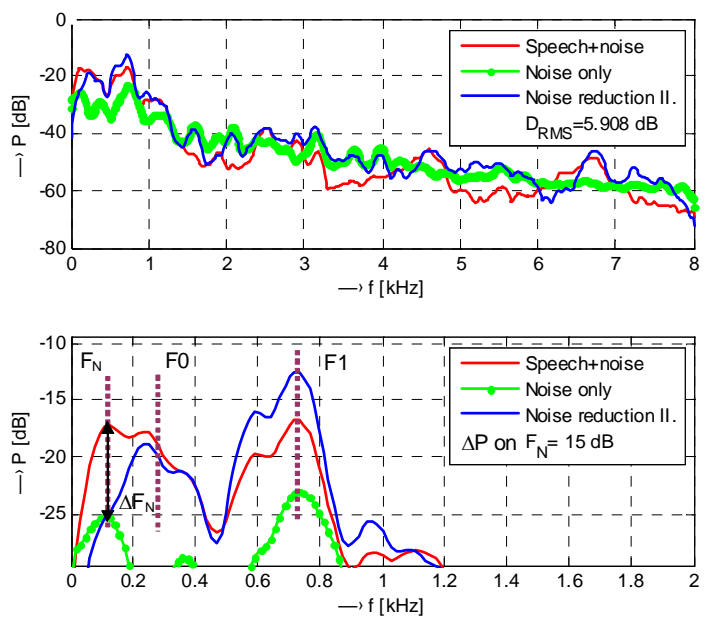

Fig.12. Welch's periodograms in $[\mathrm{dB}]$ of selected ROI from $4 \mathrm{~s}$ to $7 \mathrm{~s}$ of the speech signal used for spectrogram calculation in Fig.11: summary periodogram for full frequency range (top), detailed one with determined $F_{\mathrm{N}}, \mathrm{F} 0$ and $\mathrm{F} 1$ positions (bottom). 
Resulting mean spectral distances calculated between periodograms of vowels recorded in NMR noisy environment and cleaned by both noise reduction methods are summarized in Table 3. Calculated mean spectral differences on the $F_{\mathrm{N}}$ position for all analysed stationary parts of vowels are introduced in Table 4 (for both type of speaker gender).

\begin{tabular}{|c|c|c|c|c|}
\hline $\begin{array}{c}\text { Stationary } \\
\text { part of } \\
\text { vowel }\end{array}$ & \multicolumn{2}{|c|}{ Male voice } & \multicolumn{2}{c|}{ Female voice } \\
\cline { 2 - 5 } & method I & method II & method I & method II \\
\hline a: $D_{\mathrm{RMS}}[\mathrm{dB}]$ & 4.598 & 7.223 & 4.516 & 5.845 \\
\hline e: $D_{\mathrm{RMS}}[\mathrm{dB}]$ & 5.708 & 6.599 & 3.551 & 4.862 \\
\hline i: $D_{\mathrm{RMS}}[\mathrm{dB}]$ & 5.794 & 7.236 & 3.769 & 5.279 \\
\hline o: $D_{\mathrm{RMS}}[\mathrm{dB}]$ & 3.841 & 5.866 & 3.639 & 6.110 \\
\hline u: $D_{\mathrm{RMS}}[\mathrm{dB}]$ & 2.692 & 4.973 & 4.596 & 5.846 \\
\hline
\end{tabular}

Table 3. Results of mean spectral distances of analysed vowels $\left(D_{\mathrm{RMS}}\right.$ are calculated between periodograms of original noisy and cleaned speech signal) - male and female voice.

\begin{tabular}{|c|c|c|c|c|}
\hline $\begin{array}{c}\text { Stationary } \\
\text { part of } \\
\text { vowel }\end{array}$ & \multicolumn{2}{|c|}{ Male voice } & \multicolumn{2}{c|}{ Female voice } \\
\cline { 2 - 5 } & method I & method II & method I & method II \\
\hline a: $\Delta \mathrm{P}[\mathrm{dB}]$ & 8.469 & 16.599 & 7.844 & 15.272 \\
\hline e: $\Delta \mathrm{P}[\mathrm{dB}]$ & 9.254 & 17.085 & 8.536 & 16.426 \\
\hline i: $\Delta \mathrm{P}[\mathrm{dB}]$ & 8.024 & 14.327 & 7.375 & 13.482 \\
\hline o: $\Delta \mathrm{P}[\mathrm{dB}]$ & 11.012 & 17.632 & 7.257 & 14.001 \\
\hline u: $\Delta \mathrm{P}[\mathrm{dB}]$ & 10.005 & 16.224 & 6.343 & 12.009 \\
\hline
\end{tabular}

Table 4. Results of mean spectral differences $\Delta \mathrm{P}$ on the $F_{\mathrm{N}}$ position (calculated between periodograms of original noisy and cleaned speech signal) - male and female voice.

\section{DISCUSSION AND CONCLUSIONS}

In our first noise reduction and comparison experiment, the testing person lies on one side during the MRI scan due to the used knee RF coil (see Fig.7). But this subject position could have an influence on the produced speech posterior movement of the tongue due to gravity. This effect will be eliminated by applying the right neck coil which is currently developed (this RF coil was absent in equipment of the tested MRI machine). In that case the object can lie in supine position, as is usual in other similar experiments. As follows from the obtained results of measured noise pressure level shown in Fig.6, the maximum of $I_{\mathrm{N}}$ was below $72 \mathrm{~dB}$, therefore testing persons don't use ear phones. The used professional condenser microphones have no effect on the homogeneity of low-magnetic field $B_{0}$ of the MRI machine, which can be also mapped by special phantoms [17]. It is important, because the magnetic field homogeneity is a significant parameter of RF coils for NMR tomography [18].

The repetition time TR of the used scanning sequence in dependence of chosen other settings (FOV, slices thickness, number of sagittal images etc.) has much influence of the $F_{\mathrm{N}}$ frequency of the analysed noise signal. On the other hand, setting of echo time TE, number of averages and first of all the chosen type of NMR sequence have effect on higher frequencies of noise signal - it means, they translate changes into the $\mathrm{F} 1$ positions. The mean $F_{\mathrm{N}}$ positions were approximately $155 \mathrm{~Hz}$ in all cases, in correspondence with the used scanning sequence (chosen as the middle value between male and female voice $\mathrm{F}_{\text {mean }}$ ) - see Table 2 .

The performed experiment confirms usability of both applied noise reduction methods based on the cepstral speech model. The significant audible differences between the noisy and cleaned speech signal were observed for all processed samples. The comparison of calculated spectral distances between original noisy and cleaned speech signal shows that the spectral changes are higher for using the second method of noise reduction method. Single spectral envelope subtraction brings also parasitic effects (e.g., aliasing), therefore some filtration techniques must be subsequently applied. This problem can be successfully solved also in the cepstral domain - by limitation of real cepstrum expansion. In the case of the two microphone method, there was a problem with infiltration of speech signal to Mic. 2, which was designated only for the noise recording. After spectral subtraction, this effect was expressed in spectrum suppression also on the F1 and F0 positions (see example in Fig.11b). Determined values of F0 and F1 positions depend on the speaker vocal tract characteristics, and are not affected by the noise suppression in the speech signal. Finally, it can be said, that these two described algorithms have a much wider range of application than the particular experimental arrangement of this paper, which can be taken as an advertisement for this aspect and should seek cooperation with groups that have different recording approaches.

In the near future, the spectral analysis of harmonic noise features for different scanning sequences needs to be performed. In the next experiment we will use the listening tests for detail audio comparison of final speech signal cleared by these two methods.

\section{ACKNOWLEDGMENT}

The work has been supported by the Grant Agency of the Slovak Academy of Sciences (VEGA 2/0090/11), by the Grant Agency of the Czech Republic (GA102/09/0989) and State program SPVV no. 2003SP200280203.

\section{REFERENCES}

[1] Vampola, T., Horáček, J., Švec, J.G. (2008). FE modeling of human vocal tract acoustic: Part I Production of Czech vowels. Acta Acustica United with Acustica, 94, 433-447.

[2] Vampola, T. et al. (2008). FE modeling of human vocal tract acoustics: Part II - Influence of velopharyngeal insufficiency on phonation of vowels. Acta Acustica United with Acustica, 94, 448-460.

[3] Švancara, P., Horáček, J. (2006). Numerical modelling of effect of tonsillectomy on production of Czech vowels. Acta Acustica United with Acustica, 92 (5), 681-688.

[4] Titze, I.R. (2006). Voice training and therapy with a semi-occluded vocal tract: Rationale and scientific 
underpinnings. Journal of Speech Language and Hearing Research, 49 (2), 448-459.

[5] Laukkanen, A.M. et al. (2008). Effects of a semioccluded vocal tract on laryngeal muscle activity and glottal adduction in a single female subject. Folia Phoniatrica et Logopaedica, 60, 298-311.

[6] Leino, T. (1994). Long-term average spectrum study on speaking voice quality in male actors. In Friberg, A., Iwarsson, J. et al. (eds.) SMAC 93: Proc. of the Stockholm Music Acoustics Conference, July 28August 1, 1993. Stockholm: The Royal Swedish Academy of Music, 206-210.

[7] Sundberg, J. (1987). The Science of the Singing Voice. DeKalb, Illinois: N. Illinois Univ. Press.

[8] Malinen, J., Palo, P. (2009). Recording speech during MRI: Part II. In Proc. of the $6^{\text {th }}$ Int. Workshop Models and Analysis of Vocal Emissions for Biomedical Applications, December 14-16, 2009. Frenze University Press.

[9] Aalto, D. et al. (2011). Recording speech sound and articulation in MRI. In Proc. of the Int. Conference on Biomedical Electronics and Devices Biodevices 2011. Rome, Italy, 168-173.

[10] Bresch, E. et al. (2006). Synchronized and noise-robust audio recordings during real-time magnetic resonance imaging scans. Journal Acoustical Society of America, 120 (4), 1791-1794.
[11] Boll, S.F. (1979). Supression of acoustic noise in speech using spectral subtraction. IEEE Transactions on ASSP, 27 (2), 113-120.

[12] Martin, R. (1994). Spectral subtraction based on minimum statitistics. In Proc. of EUSIPCO 1994, 1182-1185.

[13] Vích, R. (2000). Cepstral speech model, Padé approximation, excitation, and gain matching in cepstral speech synthesis. In Biosignal 2000: Proc. of the $15^{\text {th }}$ Biennial EURASIP Conference, 77-82.

[14] Welch, P.D. (1967). The use of fast Fourier transform for the estimation of power spectra: A method based on time averaging over short, modified periodograms. IEEE Transactions on Audio and Electroacoustics, AU-15, 70-73.

[15] Stoica, P., Moses, R.L. (1997). Introduction to Spectral Analysis. Prentice-Hall, 52-54.

[16] ESAOTE S.p.A. (2008). E-scan Opera. Image Quality and Sequences Manual. 830023522 Rev. A.

[17] Frollo, I. et al. (2010). Measurement and imaging of planar electromagnetic phantoms based on NMR imaging methods. Measurement Science Review, 10 (3), 97-101.

[18] Andris, P., Frollo, I. (2002). Optimalisation of NMR coils by genetic algorithms. Measurement Science Review, 2, 13-22. 